\title{
A Collaborative Partnership between the Multicenter Handoff Collaborative and an Electronic Health Record Vendor
}

\author{
Bommy Hong Mershon ${ }^{1}$ Andrea Vannucci ${ }^{2}$ Trent Bryson ${ }^{3} \quad$ Felix Lin ${ }^{4} \quad$ Philip E. Greilich $^{3}$ Guy Dear ${ }^{5}$ \\ Patrick Guffey ${ }^{6}$ Aalok Agarwala ${ }^{7}$
}

${ }^{1}$ Department of Anesthesiology and Critical Care Medicine, Johns

Address for correspondence Bommy Hong Mershon, MD, Hopkins University School of Medicine, Baltimore, Maryland, United States

${ }^{2}$ Department of Anesthesiology, University of Mississippi Medical Department of Anesthesiology and Critical Care Medicine, Johns Hopkins University School of Medicine, 1800 Orleans St. Suite 6364, Center, Jackson, Mississippi, United States

${ }^{3}$ Department of Anesthesiology and Pain Management, University of Texas Southwestern Medical Center, Dallas, Texas, United States

${ }^{4}$ Technical Services, Epic Systems Corporation, Verona, Wisconsin, United States

${ }^{5}$ Department of Anesthesiology and Pediatrics, Duke University, Durham, North Carolina, United States Baltimore, MD 21287, United States (e-mail: bhong3@jhmi.edu).

\footnotetext{
${ }^{6}$ Department of Anesthesiology, University of Colorado School of Medicine, Aurora, Colorado, United States

${ }^{7}$ Department of Anesthesiology, Massachusetts Eye and Ear, Harvard Medical School, Boston, Massachusetts, United States
}

Appl Clin Inform 2021;12:647-654.

\section{Abstract}

Keywords

- handoffs

- collaboration

- ergonomics
Objectives The operating room is a specialized, complex environment with many factors that can impede effective communication during transitions of care between anesthesia clinicians. We postulated that an efficient, accessible, standardized tool for intraoperative handoffs built into standard workflow would improve communication and handoff safety. Most institutions now use an electronic health record (EHR) system for patient care and have independently designed intraoperative handoff tools, but these home-grown tools are not scalable to other organizations and lack vendorsupported features. The goal of this project was to create a standardized, intraoperative handoff tool supported by EHR functionality.

Methods The Multicenter Handoff Collaborative, with support from the Anesthesia Patient Safety Foundation, created a working group of frontline anesthesia experts to collaborate with a development team from the EHR vendor (Epic Systems) to design a standardized intraoperative handoff tool. Over 2 years, the working group identified the critical elements for the tool and software usability, and the EHR team designed a standardized intraoperative handoff tool that is accessible to any institution using this EHR. Results The first iteration of the intraoperative handoff tool was released in August 2019, with a second version in February 2020. The tool is standardized but customizable by individual institutions.

Conclusion We demonstrate that work on complex health care processes critical to patient safety, such as handoffs, can be performed on a national scale through crossindustry collaboration. Frontline experts can partner with health care industry vendors to design, build, and release a product on an accelerated timeline. received

January 7, 2021

accepted after revision

May 25, 2021
DOI https://doi.org/

$10.1055 / \mathrm{s}-0041-1731714$

ISSN 1869-0327. (c) 2021. The Author(s).

This is an open access article published by Thieme under the terms of the Creative Commons Attribution-NonDerivative-NonCommercial-License, permitting copying and reproduction so long as the original work is given appropriate credit. Contents may not be used for commercial purposes, or adapted, remixed, transformed or built upon. (https://creativecommons.org/ licenses/by-nc-nd/4.0/)

Georg Thieme Verlag KG, Rüdigerstraße 14, 70469 Stuttgart,

Germany 


\section{Background and Significance}

George Bernard Shaw said, "The single biggest problem in communication is the illusion that it has taken place." This quip is especially true in health care, where rapidly changing factors add complexity and increase the likelihood of errors from communication breakdowns. The Institute of Medicine defines handoffs as transitions during which responsibility for the care of a patient is transferred from one provider or team to another. Handoffs are highly vulnerable to safety failures. ${ }^{1}$ The Joint Commission reports that communication errors are the attributable root cause in $65 \%$ of sentinel events, with up to $80 \%$ of serious medical errors specifically related to miscommunication at handoff. 2,3

Complications related to anesthesia have been associated with inadequate communication since at least 1978, when poor communication was identified as the third most frequent factor associated with anesthesia errors. ${ }^{4}$ The intraoperative period is particularly challenging for handoffs because (1) experience and level of training often differ among anesthesia providers, (2) the point in the procedure affects the type and amount of information that needs to be relayed, and (3) real-time patient care issues can arise during handoffs that require attention and intervention by the anesthesia providers. Patient deterioration and constant monitoring of alarms require clear and complete communication to resolve. However, communication can be complicated by the noisy, sometimes chaotic, nature of the operating room. Consequently, information quality and completeness can be affected. The intraoperative handoff is unique as compared with other perioperative handoffs. Postoperative handoffs naturally occur once surgery has been completed, the patient has been stabilized and all intraoperative information has been collected. The postoperative environment is usually more controlled allowing providers to focus on the handoff itself, which alleviates some of the difficulties faced during an intraoperative handoff. The anesthesia provider also is fully transferring patient care to the next team, either to recovery room nurses or an intensive care unit team of doctors and nurses. This differs from the intraoperative handoff when handoffs occur from an anesthesia provider to an anesthesia provider at different points of the surgery in real time.

Quality improvement and lean methodology are helpful when designing an effective and useful handoff process. A handoff process that is standardized and embedded into routine workflow can improve patient safety by decreasing waste and improving efficiency. ${ }^{5}$ Standardization reduces complexity by making elements of care routine. ${ }^{6}$ For example, standardizing central venous catheter insertion has significantly reduced the incidence of central line infections, ${ }^{7}$ and insulin administration protocols have decreased medication errors and hypoglycemic episodes. ${ }^{8}$ Intraoperative handoffs also can benefit from a structured approach. Evidence-based best practice for improving team-based communication supports implementation of a structured handoff process with cognitive aids, such as checklists. ${ }^{9}$ But published studies are few in number and the developed checklists are most often specific to the authors' institution. ${ }^{10}$

The widespread adoption of electronic health record (EHR) systems presents an opportunity to decrease waste, increase efficiency, and augment standardization by incorporating handoff checklists into routine processes within the EHR. EHR-based cognitive aids have the potential to increase reliability of information transfer by combining real-time patient-specific clinical data with a standardized handoff in a digital format. However, to date, no published reports have described an intraoperative handoff intervention that can be (1) implemented across all types of anesthesia information systems, (2) standardized with important handoff elements but customizable to the nuances of an institution, and (3) fitted within the ergonomics of the intraoperative workflow.

In September 2017, the Anesthesia Patient Safety Foundation (APSF), in full support of the Multicenter Handoff Collaborative (MHC), hosted a perioperative handoff consensus conference ${ }^{11,12}$ to address handoffs at a national level. Key findings were that standardized tools could help facilitate communication during handoff and that EHR systems should be used to help when possible. To build better tools that could be implemented within the EHR, the MHC formed a partnership with Epic Systems (Verona, Wisconsin, United States), an EHR software developer and vendor, to accelerate development of EHR-based tools that could facilitate information transfer during perioperative handoffs. This crossindustry partnership accelerated the design and implementation of a durable, adaptable intraoperative handoff tool that was made widely available to all Epic Systems users.

\section{Methods}

\section{MHC Formation}

In 2015, a group of physician anesthesiologists from several large U.S. academic hospitals met to discuss their individual efforts to build and implement tools to assist with intraoperative anesthesia handoffs within the Epic EHR system. Although content varied from institution to institution, struggles with creation, implementation, and compliance were universal. The need for tools designed and supported by the EHR software became a common theme of discussion.

\section{MHC EHR Workgroup Formation}

With the support of APSF, the MHC expanded to form different workgroups, such as the EHR workgroup. This group included representation from Johns Hopkins University, Massachusetts General Hospital/Harvard University, University of Texas Southwestern - Dallas, University of Colorado/Children's Hospital Colorado, Duke University, University of Mississippi, and University of Rochester.

\section{Collaborative Partnership between EHR Workgroup and Epic EHR}

After the initial meeting, this group contacted members of Epic's physician specialty steering board, a group of physicians who help to direct EHR content and development for each specialty and advocate for support to improve the end-user handoff experience. When the board agreed that a handoff tool was needed, Epic assigned a team to meet with representatives from the seven different institutions. 
2015
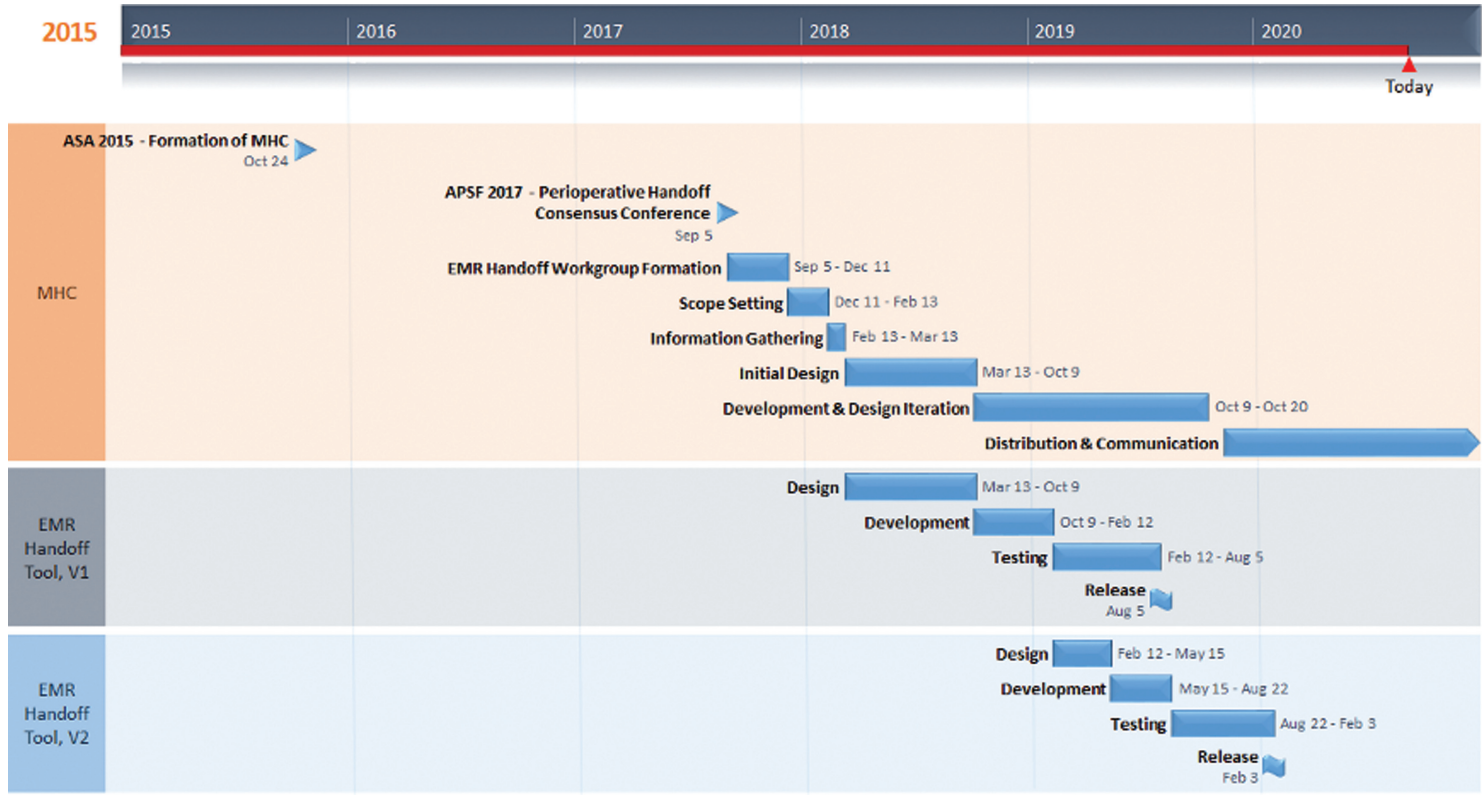

APSF 2017 - Perioperative Handof Consensus Conference EMR Handoff Workgroup Formation $\square$ Sep 5 - Dec 11 Scope Setting Dec 11 - Feb 13 Information Gathering Feb 13 - Mar 13 Initial Design $\square$ Mar 13-Oct 9 Development \& Design Iteration oct 9 - Oct 20 Design $\square$ Mar13-Oct9 Development $\square$ oct 9 - Feb 12 Testing Release
Aug 5

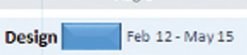

Development $\square$ Moy 15 - Aug 22 Testing $\quad$ Aug 22 - Feb 3 Release
Feb 3

Fig. 1 Timeline of MHC formation, EHR workgroup, and Epic Systems partnership from development and dissemination of the intraoperative handoff tool in the EHR system. EHR, electronic health record; MHC, Multicenter Handoff Collaborative.

The first meeting between the MHC EHR workgroup and Epic took place in December 2017 and focused on shared discovery of the tools in use at the various hospital systems. After comparing the earlier attempts and examining local feedback on each, the initial design approach was established. The intent was to reduce much of the clutter common in many of the tools, and to streamline an overview of important patient factors, with links to more detailed information as needed for more efficient and quicker viewing of necessary information. ${ }^{13,14}$ Monthly meetings were scheduled to explore and develop content to satisfy common user requirements. Verbal and written surveys were conducted over several months to reach consensus on critical handoff elements. In subsequent meetings, members discussed various design and workflow decisions, including click reduction and incorporation of other mandatory documentation. The work timeline spanned from the September 2017 APSF conference through the August 2019 distribution of the first handoff tool in Epic (-Fig. 1).

Once the goals were established, the vendor conducted a gap analysis between the existing EHR functionality and the functionality envisioned for the final product. These gaps were combined with an estimate of programming time to generate a project development list. Over 7 months, the Epic created a workflow and built a report that met most of the group's requests. The report was then subjected to the EHR's routine internal quality assurance systems, including prototype build, end-user testing, two rounds of code review, two rounds of internal testing, steering committee and internal leadership review, and a severalmonth holding period to validate stability and performance.

\section{Results}

The initial version of the handoff tool ( - Fig. 2) was available in August 2019 and an updated version (-Fig. 3) in February 2020. Given that various Epic customers are on different

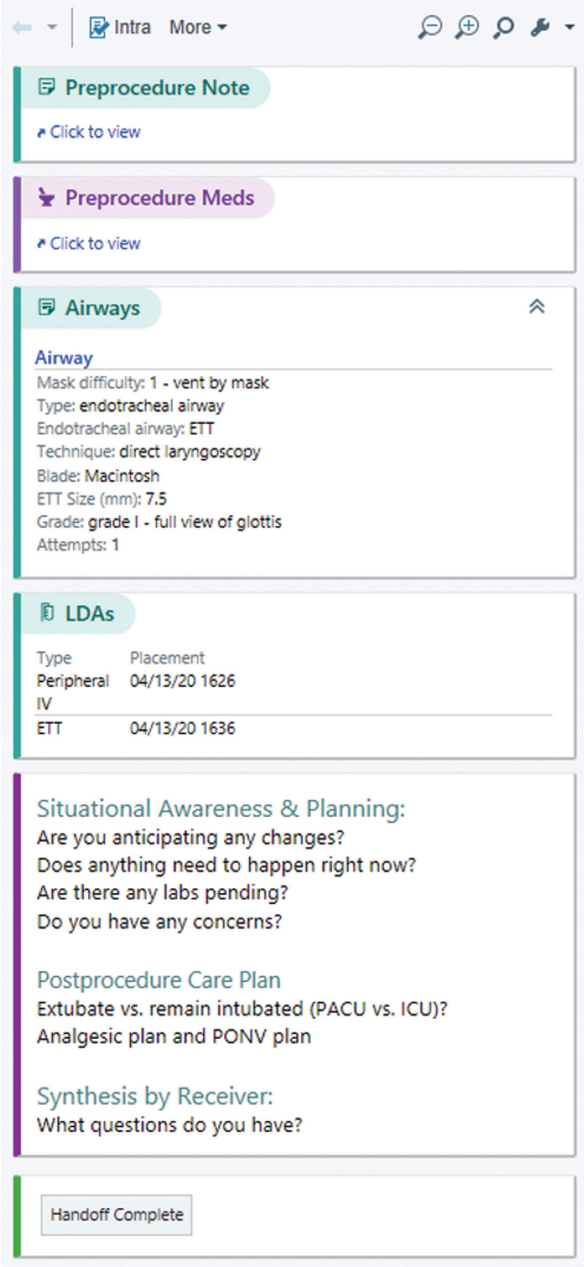

Fig. 2 First version of the intraoperative handoff tool (reprinted with permission from (C2020 Epic Systems Corporation) in the Epic intraoperative record. Limitations related to programming in Epic prevented the complete inclusion of all elements that the group had requested during initial planning. 


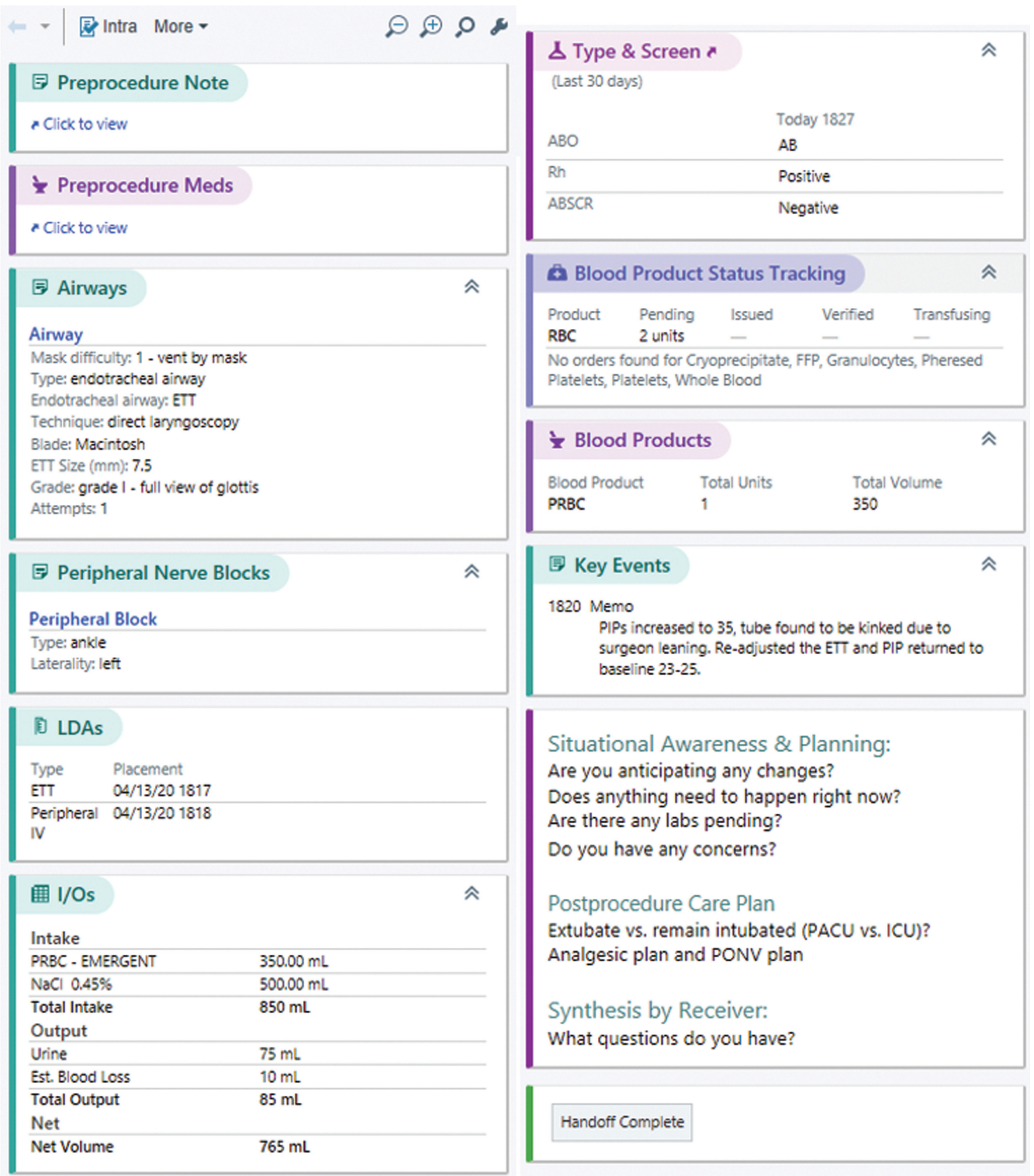

Fig. 3 Current updated version of the intraoperative handoff tool (reprinted with permission from @2020 Epic Systems Corporation) in the Epic intraoperative record. It includes more critical information elements that our group agreed were necessary. In the live intraoperative record, this sidebar is shown as a continuous vertical column that can be viewed by scrolling up and down. The goal is to limit to one scroll to view the entire tool. When an anesthesia provider clicks the Handoff Complete button at the end, the staffing grid will automatically open to allow the provider to be able to sign over to the next provider at the time of the handoff. It also inserts the handoff icon into the intraoperative record to mark the time of the handoff.

upgrade cycles, the vendor anticipated that the tool would be available to approximately $50 \%$ of their customer base by August 2020 and to most customers by February 2021.

\section{Handoff Tool Content in Epic}

Critical elements included medical history, surgical procedure, airway details, intravenous line details, fluid balance (including blood products), medication allergies, intraoperative medications, relevant laboratory data, and unexpected events. Much of this detail already existed in other portions of the intraoperative documentation window and patient header. To avoid redundancy and minimize switching between screens, the group decided to build a handoff report within the sidebar of the main screen. This configuration allowed users to continue seeing the patient management information within the patient header and other portions of the screen, along with the active intraoperative grid of medications and the graphical vital signs.

\section{Layout of Handoff Tool Content in Epic}

To maximize efficient use of screen space and minimize scrolling, ${ }^{14}$ certain information elements such as the Preprocedure Note and Preprocedure Meds were selected for access via hyperlinks rather than displayed completely within the sidebar (-Figs. 2 and 3 ).

The Preprocedure Meds section was configured to show only active home medications from the previous 14 days and active inpatient medications administered during the previous 7 days, with a focus on medications administered preoperatively. Other sections were streamlined to display 
only information most pertinent to an anesthesia provider, rather than the full procedure notes.

The LDA (Lines, Drains, and Airways) section displays the invasive devices present in the patient at the moment of the handoff, with date and time of their placement, including:

- Intravenous lines: peripheral intravenous, central venous catheters.

- Drains: urinary catheter, spinal fluid drain.

- Airways: endotracheal tube, supraglottic airway (laryngeal mask airway or other types).

Next, the Discussion Prompts section reminds anesthesia providers to share clinical judgment and information about the patient's status that may not be captured in the objective EHR data.

Finally, at the bottom of the report is a button to mark Handoff Complete. Clicking this button (1) files the "Handoff Event," marking the formal transition of care and (2) opens the "Staffing" page, where formal documentation of the new anesthesia provider(s) is completed. This important feature was universally requested by group members because it assists clinicians in completing required documentation more efficiently with fewer clicks than was previously possible.

The latest version ( - Fig. 3 ) includes additional elements such as procedure notes for peripheral nerve block placement, patient blood type, blood products administered, total fluid balance accounting for input and output, and other key events that may have occurred during the surgical procedure. Importantly, the Key Events section was designed to be customizable so that specific events and quick notes deemed important by an institution could be extracted. The development of these sections is especially notable as the standard intraoperative chart does not easily show this information. All linked and displayed informational elements were chosen to keep the display as short as possible and support the cognitive function of anesthesia providers without overwhelming them.

Epic software allows the creation of a dedicated report to track use of this tool within each organization. This report can be customized to monitor utilization by specific groups of anesthesia providers such as attending anesthesiologists, anesthesia fellows and residents, certified registered nurse anesthetists (CRNAs), anesthesia assistants (AAs), and others.

\section{Customization of Handoff Tool}

The handoff tool provides a structured approach, incorporates the prompting features of a checklist, and supports documentation of compliance as well as an intuitive and streamlined clinical workflow. However, to encourage individual institutional adoption, several elements were designed to be customizable:

- Handoff buttons specific to staff type (attendings, fellows, residents, CRNAs, AAs).

- Attestation triggers specific to staff type (attendings, fellows, residents, CRNAs, AAs).

- Reminders for controlled substance reconciliation, an important regulatory focus.
- Integration of mnemonics that might be used at a specific institution.

- Reporting to track use of the handoff workflow (i.e., number of handoffs that have occurred).

The many possibilities for customization are too numerous to list. Therefore, we present examples of what can be done to illustrate how an institution can customize the handoff tool. The anesthesia team at the Massachusetts General Hospital retained the general structure of the report but made the following changes:

- Added the staff handoff button to the top of the report.

- Updated the text of the button to be more descriptive: "Document Handoff Event, Launch Staff Activity. and Close Report."

- Added procedure information to the report (procedure, diagnosis, surgeon, etc.).

- Pulled text from a free-text "anesthesia day of surgery notes" field on the preop note directly into the report.

- Added displays for the allergies, problem list, medical history, and anesthesia-related family history in the patient's chart to the report.

- Included details on all the lines, drains, and airways in the report.

- Added links to any imaging from the past 5 days.

- Added the most recent result for a selection of 25 laboratory components that they deemed important to display.

Our goal for this project was to develop an end product that could be deployed at most of several hundreds of health care organizations that use Epic Anesthesia, which range from small community hospitals to large, multihospital academic health systems. Therefore, we tried to balance three factors-comprehensiveness, brevity, and ease of implementation. Ideally, we desired a comprehensive tool that could be used for teaching at academic centers as well as a brief tool that an experienced anesthesiologist taking care of a low acuity patient would not find cumbersome, and an easy-to-implement tool at a small, community hospital with limited resources to customize. Based on these competing priorities and the experiences of our workgroup members, creating a single, out-of-the-box solution that was ideal for every health care organization was not realistic. Instead, our approach (which aligned with Epic System's philosophy) was to create a standard framework shared by everyone with a basic tool optimized for an average community health care organization, with a flexible array of configuration options that allowed large, academic institutions to build upon that base and optimize for their workflow. The Massachusetts General Hospital is a large teaching institution with skilled clinical and information technology (IT) resources that could be devoted to creating a more complex and comprehensive customized tool according to their preferences.

\section{Future Developments}

The tool is currently available on the Epic desktop configuration, but not yet on Epic mobile platforms developed for smart phone and tablet use such as Haiku and Canto. 
Developers continue to improve and add important elements that have been designated as crucial to improving the handoff process, such as:

- Support of an operating room-to-recovery room/intensive care unit handoff report.

- Intraoperative medications with last dose, last dose time, and total dose.

- Reporting and transferring of controlled substances.

- Inclusion of checklists specific to different intraoperative contexts (cardiac surgery, neurosurgery, labor and delivery, etc.).

- Improved LDA information, including the use of color coding or an avatar to help identify the location of lines.

- Improved mobile version for mobile devices.

- Forcing function for handoff attestations and/or filing handoffs.

\section{Discussion}

Both local patient safety champions and EHR vendors have been frustrated by the lack of progress in creating a usercentric EHR-based cognitive aid that can be widely used for intraoperative handoffs. The difficulty may be due in part to the complexity of designing a durable solution, which requires a level of expertise and capability that does not reside in any single organization (hospital or vendor). Here, we show that collaboration between a special interest group (SIG) and an EHR vendor can be used to create a widely accessible solution. Members of the MHC EHR Working Group provided expertise from around the country to work with the Epic design team to balance end-user needs with software constraints. The support of the vendor's project team was instrumental in coordinating and navigating the design process and ultimately producing a viable solution. In exchange, the vendor benefitted from having a "brain trust" to establish user requirements for a single solution, as opposed to fragmented local solutions. Going forward, we will disseminate user requirements to make them accessible to other vendors and health systems. Continued partnership will allow us to assess adoption, feedback, and improvements to the intraoperative cognitive aid, and create EHR-based aids for other types of perioperative handoffs, such as those between the operating room and postanesthesia care unit or intensive care unit. We are also disseminating this work to other interprofessional societies and at national and international meetings.

The ability to accelerate the development of scalable, durable solutions to complex patient safety priorities will likely require innovative approaches to the ergonomics of the change process. Although our group found success by bringing clinicians and EHR developers together with the guidance and support of APSF, additional work will be needed if these types of innovative, cross-industry partnerships are to be generalizable. We believe clear delineation, dissemination, and alignment of patient safety priorities must be the central focus to each group's strategy. For example, APSF has clearly outlined its 12 patient safety priorities ${ }^{15}$ and has begun providing initial support through guidance, networking, consensus building, and seed funding for SIGs like the MHC. Time and effort by context experts or clinicians remain the most fragile aspect of this approach. The reliance on extramural funding of academic physicians and potential for publications is unreliable and not translatable to most private or academic settings. The value and return on investment depend on a group's alignment with the partnering organization's strategy, whether it is a health system, large private practice group, national medical society, or vendor. Other examples of success using this innovative macroergonomic technique include the Michigan Keystone project $^{16}$ and Safe Surgery program in South Carolina. ${ }^{17}$

\section{Limitations and Challenges}

Our approach had several limitations. First, the MHC EHR Working Group is a relatively small group of anesthesiologists who work under significant time constraints. The development of platforms to facilitate asynchronous work would have increased this group's efficiency. Second, the solution produced by this effort may not be completely generalizable given the differences in constraints of software from other vendors. Third, the tool may have been improved by including representatives from nonacademic sectors (private practice, community hospitals, etc.) and other anesthesia providers (CRNAs, AAs, residents, or fellows). Lastly, access to the most current version of the EHR-based cognitive aid for intraoperative handoffs will be based an institution's upgrade cycle.

One broad challenge that Epic developers faced in designing the software was creating technical frameworks that were both flexible and optimized. Producing results on a reasonable timeline necessitated many complex tradeoffs between creating tools that were universal enough to apply to most institutions and tools that were effective enough to drive adoption by individual providers in their unique practice environments. Limitations related to programming in Epic prevented inclusion of all elements that the group had requested during initial planning. Epic developers focused on creating a tool that presented available elements in the most user-friendly format for easier adoption by more institutions, rather than including elements in a suboptimal format with extraneous information.

A limitation of the current tool is the absence of a function to force compliance and to demonstrate that both anesthesia providers reviewed the handoff tool and, more importantly, were actively engaged in the handoff process. Without such a "hard stop," it is possible for the receiving clinician to bypass all the information and cognitive steps that we designed.

The need for flexibility arose from the significant variation in optimal content and format of a handoff tool, which depends on the current procedure, recent and ongoing plan of care, patient demographics, patient medical history, practice model, institutional and EHR workflows, and EHR configuration. Additional variation came from the lack of a universal, research-backed structure for perioperative handoffs, equivalent to SBAR (Situation-Background-AssessmentRecommendation) or IPASS (Illness severity, Patient summary, Action list, Situation awareness, Synthesis by receiver), 
that could be used as standard scaffolding. In the absence of standard practice, digital or otherwise, optimization was essential. A new tool needed to be compelling enough to encourage providers to adopt both it and the associated workflow. Because time available to physician members and Epic programmers was limited, and we wanted to produce results within a few years, we could not realistically aim for an ideal product that could be released at once. Instead, we started with that ideal, but then identified the most efficient path to move from the current state to a product whose quality was sufficiently high to merit release using an iterative quality improvement process.

One example of this development process is the strategy for displaying laboratory results. The ideal would be to display only meaningful results. However, the definition of what is meaningful is not straightforward. For instance, liver function tests would not be of particular concern during a perioperative handoff unless the patient had a history of liver disease or the values were unexpectedly abnormal or trending in the wrong direction. They might also be important if acetaminophen administration was planned for a patient with abnormal laboratory results. While all of this is technically possible, the time required to program algorithms for all possible laboratories and all possible scenarios would be overwhelming, and further complicated by the fact that (1) there is no concrete, standard criteria for what is meaningful, and significant flexibility would be required for each institution to customize the criteria and (2) there is no standard crosswalk between the mnemonics used by various laboratory information systems for laboratory components and the clinical concepts they represent; therefore, each institution would need to devote IT staff and physician time to generating that crosswalk.

Taking into account these challenges, we ultimately chose to include only the patient's most recent blood type and antibody screen $(T+S)$. The workgroup held broad consensus that the most recent $\mathrm{T}+\mathrm{S}$ results are critical and that only the most recent results matter. Moreover, because the laboratory consists of relatively few components, it was a reasonable choice for inclusion. Thus, we could release a relatively consistent and minimal display format for this information, and it would be a reasonable lift for each institution to map its unique laboratory component mnemonics onto our standard display format.

\section{Success of Collaborative Partnership}

A major barrier to addressing complex patient safety priorities is the lack of alignment and working relationships between clinicians, subject matter experts, and patient safety organizations. For SIGs like the MHC to be effective, national partners must set clear priorities and sponsor groups working to drive the creation of durable solutions. In this case, leaders from the MHC and APSF supported the APSF's priority to improve care transitions by working together in a consensus conference on the issue and bringing together stakeholders from multiple organizations to raise awareness, develop working relationships, and encourage efforts for improvement. The early success in designing a widely available EHR-based cognitive aid has helped to ensure continued collaboration to track adoption and improve the product. Our future goals are to measure implementation, use, and effectiveness of the handoff tool and to design and improve other perioperative handoff processes.

\section{Conclusion}

The absence of a structured approach to intraoperative handoffs that uses an EHR-based cognitive aid for every handoff undermines our ability to reduce/eliminate medical errors and harm to patients. With the support of a national organization, our innovative cross-industry partnership aligned context and content experts to develop an accessible, customizable solution for Epic users within a 2-year period. This partnership was the key factor that accelerated the design and seamless integration of a new cognitive aid with existing, required handoff documentation workflow in the EHR. We were also able to streamline display of information elements in the most efficient, relevant, and useful way for anesthesia providers. In future steps, we plan to improve the representation of our working group, design durable solutions for other perioperative handoffs and care transfers, and advocate for durable strategies that ensure access for busy clinicians to serve as context experts and participate in SIGs.

\section{Clinical Relevance Statement}

Effective communication during handoffs during surgical procedures is challenging due to the complex environment, multiple distractions, and intraoperative handoffs that occur while the procedure is in progress. Practicing anesthesiologists with a background in quality, safety, human factors, and informatics determined via a consensus process the critical elements for a successful handoff. Members of this team collaborated with the software developers from an EHR to develop, test, and deploy a standardized and locally customizable handoff tool to all of the vendor's hospitals and health systems.

\section{Multiple Choice Questions}

1. Which of the following are benefits of using a collaborative approach between subject matter experts and electronic health record vendors to improve clinical processes?

a. Vendors benefit from clinical expertise to improve their products

b. Clinicians benefit by improvements to workflow and reduced administrative burden

c. Patients benefit by potential improvements in safety d. All of the above

Correct Answer: The correct answer is option d. Collaborative partnerships between experts of different industries to design solutions for complex health care processes such as perioperative handoffs allow subject matter experts to develop a shared mental model of (1) how 
each expert's work environment operates and (2) identify elements that are necessary and/or feasible for that workspace. If clinicians know what an ideal electronic handoff tool should look like but have little to no knowledge of the limitations of the electronic information system they are using, then the handoff tool that is developed can be not useful for their workflow. It is also important for the developers and programmers to understand what clinicians desire to build an optimal tool or program that functions well within the workflow. Building a clinical solution that fits within the capabilities of the electronic information system as well as the demands of the clinical workflow will result in a product that is well-designed, usable, and helpful for the clinician, which in turn can improve patient care, quality, and safety.

2. A primary challenge in designing and creating an EHR tool is

a. Difficulty identifying critical elements for handoff communication

b. Lack of accurate information within the electronic health record

c. Creation of frameworks that are both standardized and locally customizable

d. Integrating both clinical information and required documentation functions into a single workflow

Correct Answer: The correct answer is option c. Ideally, an electronic tool to help with health care processes needs to have a standard framework that is considered essential, regardless of the location or size of the health care organization. But to accommodate and allow for differences in workflow and resources based on location and size of the health care organization, the tool must also be flexible to allow for customization builds in content and design to be optimal for the specific needs of that particular clinical environment. For example, within anesthesia practice, certain elements of an intraoperative handoff are considered essential, regardless of where an anesthesia provider practices or what types of cases he/she provides anesthesia for or the level of experience of the anesthesia provider. However, where an anesthesia provider practices and/or the primary scope of practice of the anesthesia provider (i.e., obstetric anesthesia, cardiac anesthesia, neurosurgical anesthesia, orthopedic anesthesia) can affect what additional elements are required and/or how the information is laid out within the electronic tool.

Protection of Human and Animal Subjects

No human subjects were involved in this project.

Funding

None.

\section{Conflict of Interest}

Author F.L.'s work documented in this manuscript was performed as part of his employment at Epic Systems. All other authors report no conflict of interest.
Acknowledgments

The authors would like to thank Claire Levine, Scientific Editor, Department of Anesthesiology and Critical Care Medicine, Johns Hopkins University, and Joanna Abraham, Assistant Professor, Department of Anesthesiology and Institute for Informatics, School of Medicine, Washington University in St. Louis.

\section{References}

1 Institute of Medicine (US) Committee on Quality of Health Care in America. Crossing the Quality Chasm: A New Health System for the 21st Century Washington, DC: National Academies Press; 2001

2 Joint Commission Center for Transforming Healthcare releases targeted solutions tool for hand-off communications. Jt Comm Perspect 2012;32(08):1

3 Jurevvicz K, Alfred M, Neyens DM, Catchpole K, Joseph A, Reeves ST. Investigating intraoperative and intraprofessional handoffs in anesthesia. Proc Hum Factors Ergon Soc Annu Meet 2018;62(01): 469-473

4 Cooper JB, Newbower RS, Long CD, McPeek B. Preventable anesthesia mishaps: a study of human factors. Anesthesiology 1978; 49(06):399-406

5 Scoville R, Little K. Comparing Lean and Quality Improvement. IHI White Paper Cambridge, MA: Institute for Healthcare Improvement; 2014

6 QuisenberryE.How doesstandard work lead to better patient safety? AccessedJune 15,2021 at: https://www.virginiamasoninstitute.org/ how-does-standard-work-lead-to-better-patient-safety/

7 Berenholtz SM, Pronovost PJ, Lipsett PA, et al. Eliminating catheter-related bloodstream infections in the intensive care unit. Crit Care Med 2004;32(10):2014-2020

8 Rozich JD, Howard RJ, Justeson JM, Macken PD, Lindsay ME, Resar $\mathrm{RK}$, et al. Standardization as a mechanism to improve safety in health care. Jt Comm J Qual Saf 2004;30(01):5-14

9 Agarwala AV, Firth PG, Albrecht MA, et al. An electronic checklist improves transfer and retention of critical information at intraoperative handoff of care. Anesth Analg 2015;120(01):96-104

10 Barbeito A, Agarwala AV, Lorinc A. Handovers in perioperative care. Anesthesiol Clin 2018;36(01):87-98

11 Agarwala AV, Lane-Fall MB, Greilich PE, et al. Consensus recommendations for the conduct, training, implementation, and research of perioperative handoffs. Anesth Analg 2019;128(05): e71-e78

12 Multiple. APSF Newsletter: The Official Journal of the Anesthesia Patient Safety Foundation. Anesth. Patient Saf. Found 2017; Vol. 32, No. 2, 29-56. Accessed June 15, 2021 at: https://www.apsf. org/wp-content/uploads/newsletters/2017/Oct/pdf/ APSF201710.pdf

13 Martinez DA, Mora E, Gemmani M, Zayas-Castro J. Uncovering hospitalists' information needs from outside healthcare facilities in the context of health information exchange using association rule learning. Appl Clin Inform 2015;6(04):684-697

14 Brown PJ, Marquard JL, Amster B, et al. What do physicians read (and ignore) in electronic progress notes? Appl Clin Inform 2014;5 (02):430-444

15 APSF. APSF's perioperative patient safety priorities. apsforg 2018. Accessed November 12, 2020 at: https://www.apsf.org/patientsafety-initiatives/

16 Reames BN, Krell RW, Campbell DAJr, Dimick JB. A checklist-based intervention to improve surgical outcomes in Michigan: evaluation of the Keystone Surgery program. JAMA Surg 2015;150(03): 208-215

17 Haynes AB, Edmondson L, Lipsitz SR, et al. Mortality trends after a voluntary checklist-based surgical safety collaborative. Ann Surg 2017;266(06):923-929 